\title{
HIGH PRESSURE PHYSICS AND CHEMISTRY IN GIANT PLANETS AND THEIR SATELLITES ${ }^{+}$
}

\author{
D.J. Stevenson \\ Division of Geological and Planetary Sciences, Califomia Institute of \\ Technology, Pasadena, CA 91125, U.S.A.
}

\begin{abstract}
Résumé - Les couches profondes de Jupiter, Saturne, Uranus, Neptune ainsi que leurs satellites sont étudiēs. Des résultats importants concernant la Physique de la Matière Condensée sont exposés. La discussion porte surtout sur $\mathrm{H}_{2}, \mathrm{H}-\mathrm{He}, \mathrm{H}_{2}-\mathrm{H}_{2} \mathrm{O}, \mathrm{H}_{2}-\mathrm{CH}_{4}, \mathrm{NH}_{3}$, et $\mathrm{CH}_{4} \cdot 5.75 \mathrm{H}_{2} \mathrm{O}$. La Planétologie bénéficiera des développements, présents et futurs, théoriques et expérimentaux.
\end{abstract}

Abstract - The deep Interiors of Jupiter, Saturn, Uranus, Neptune, and their satellites are discussed. The important issues relevant to the physics of condensed matter are identified. The discussion emphasizes $\mathrm{H}_{2}, \mathrm{H}-\mathrm{He}, \mathrm{H}_{2}-$ $\mathrm{H}_{2} \mathrm{O}, \mathrm{H}_{2}-\mathrm{CH}_{4}, \mathrm{NH}_{3}$, and $\mathrm{CH}_{4} \cdot 5.75 \mathrm{H}_{2} \mathrm{O}$. Present and future theory and experiment are of great benefit to planetary sclence.

The last decade has seen drametic developments in our understanding of planets, primarily because of the data returned by deep space missions. For example, the Voyager mission consists of two spacecraft which flew past both Jupiter and Saturn; one of those spacecraft will be the first man-made object to visit Uranus, In early 1986. One of the ultimate goals of this exploration effort is to elucidate the origin and composition of the solar system. This requires both spacecraft data and a knowledge of the properties of relevant materials at the thermodynamic conditions encountered within the planets and their satellites. The high pressure physics community is providing an essential service to planetary science in this area. My goal in this brief summary review is to outilne the present state of affairs and identify the areas where present and future experiment or theory can provide substantial advances.

A detailed determination of the internal properties of planets can never be achieved except by direct sampling. The interpretation of planetary interiors must rely heavily on "models" which simplify, by judicious cholce of assumptions, the complex nature of real planets. For our present discussion, the two most important assumptions deserving further discussion are those concerning composition and the internal distribution of constituents. (Other assumptions, such as hydrostatic equilibrium, are crucial to the models but are of well-established validity and merit less concern.)

The bulk compositions of glant planets and their satelites are assumed to be related to cosmic abundances (or, almost equivalently, the primordial composition of the sun). The relationship is not an identity since planetary composition is also determined by relative volatility (1.e. which constituents can condense under the thermodynamic conditions encountered during planetary formation). It is convenient to subdivide the constituents into three classes of materials: gases, ices, and rock. 'Gases' mean primarily hydrogen and helium,

\footnotetext{
+ Contribution number $\mathbf{4 1 4 3}$ of the Division of Geological and Planetary sciences, California Institute of Technology, Pasadena, California 91125.
} 
which comprise 0.92 and 0.08 (approximately) number fraction of a cosmic mixture. All other elements combined comprise $-10^{-3}$ number fraction. Although these elements are overwhelmingly most abundant, they are also the most volatile and can only be incorporated into a planet in gaseous form. High volatility and high molecular velocity (implying ease of escape) prevents these constituents from being significant components on bodies \ Earth in size. Not surprisingly, however, hydrogen and helium are the main constituents of Jupiter and Saturn. The "ices" mean $\mathrm{H}_{2} \mathrm{O}, \mathrm{CH}_{4}, \mathrm{NH}_{3}$, and possibly $\mathrm{CO}, \mathrm{CO}_{2}, \mathrm{~N}_{2}$; materials which form from $\mathrm{O}, \mathrm{C}$, and $\mathrm{N}$; respectively the third, fourth, and sixth most abundant elements in the Universe. (The fifth most abundant element is Ne and is a very minor component of 'gases'). The "'ices"' are capable of condensation (as solids) in the outer solar system, to varying degrees depending on distance from the Sun. Water 1ce appears to predominate at Jupiter; the other ices are present (but in uncertain amounts) in more distant bodies. The planets Uranus and Neptune are predominantly icy. The third class of material is "rock"', essentially everything other than "gases"' and "ices." The primary constituents of "rock' are those found in the Earth: magnesium silicates and iron (both as metal and bound up in silicates or oxides).

When models of planets are constructed, it is usual to treat these three material classes as the primary building blocks and to assume (with minor variations) that the relative elemental abundances within a material class are equal to the cosmic abundances. For example, the $\mathrm{C}: 0$ ratio might be kept fixed but the H:O ratio might be an adjustable parameter. It is important to understand that this procedure is an assumption. We do not understand enough about the early solar system, or the dynamics and phase mixing of planetary interiors to assess with confidence the validity of this approach. Nevertheless, it yields results consistent with observable properties. An obvious corollary of this approach is that planets and perhaps satellites are layered, in accordance with the relative densities of the three constituent classes: rock core, ice layer, gas envelope. Although this gives the lowest (1.e. most negative) gravitational energy, it is not necessarily the preferred thermodynamic state if the layers are miscible (e.g. if the "ice" can mix fully with the "gas"'). It is in this area, in particular, that our knowledge is most limited and where future experiment and theory will help immensely.

It is not possible to describe the construction of planetary models here. The interested reader will find a brief review [1] or more detailed discussions $[2,3,4]$ elsewhere. For our present purpose, it is most useful to list the thermodynamic conditions encountered:

Table I

Thermodynamic Conditions in Planets and Satellites

$\begin{array}{lcc}\text { Body } & P_{\text {center }} \text { (Mbar) } & T_{\text {center }}(\mathrm{K}) \\ \text { Large Satellites } & \sim 0.05 & \sim 1600 \\ \text { Mercury, Mars } & -0.4 & \sim 2500 \\ \text { Earth, Venus } & -3 & \sim 4500 \\ \text { Uranus, Neptune } & \sim 10 & \sim 8000 \\ \text { Saturn } & \sim 30 & 12000+ \\ \text { Jupiter } & \sim 100 & 20000+\end{array}$

Pressures are obtained from solution of the equation of hydrostatic equilibrium with given assumptions for the constituents and their layering. Temperatures are 
obtalned from knowledge of the internal heat flow (measured for Earth, Jupiter, Saturn, and Neptune; inferred for the others) and theories of convection (since all planets and satellites eliminate heat primarily by convection - regardless of whether they are solid or fluid). We will now discuss brlefly each body (or class of body) in the outer solar system, in order of decreasing mass.

Jupiter is arguably the best understood planet in our solar system (including Earth). All known properties are consistent with a body that consists of $\sim 95 \%$ cosmic abundance mixture (by mass), w1th the remaining $\sim 5 \%$ (1.e. ten-twenty Earth masses) in the form of a dense (probably rock) core. Of the cosmic envelope, roughly $70 \%$ is a metallic hydrogen-helium-minor constituent mixture. The outermost $30 \%$ is a molecular hydrogen-helium-minor constituent mixture. The amount of ice might be in excess of cosmic abundance since direct observations of the atmosphere indicate a factor of two enhancement of methane. The temperature within Jupiter is high enough that no first order phase transitions (other than cloud condensation of minor constituents) are encountered at $P \leqslant 1$ Mbar, and it is unclear whether molecular $\rightarrow$ metallic hydrogen is a first order transition or immiscibility of helium occur at still greater depths (these issues are discussed further below).

Saturn is superficially rather similar to Jupiter, but with some important differences. It has a higher mass fraction $(\sim 20 \%)$ in the form of dense (rock?) core and IR data suggests that it has a helium depletion (by factor of two relative to cosmic) in the outer envelope. Saturn's lower mass also means that a much smaller fraction ( one third) of its mass is a metallic hydrogen-dominated mixture. The helium depletion suggests limited solubility of helium in hydrogen (discussed further below).

Uranus and Neptune are much less well understood but are clearly very different from Jupiter and Saturn. A consistent model has a rock core of several Earth masses surrounded by a water-rich layer which extends part way or all the way to an atmosphere that is known to be hydrogen-dominated. It is not known whether there is an abrupt or gradual transition from water-dominated conditions, although it is argued below that an abrupt ("ocean" $\rightarrow$ atmosphere) transition is not required. In any event, the ice component of these planets is typically $\sim$ half the total mass, but with a large uncertainty.

Large satellites range from Io and Europa (rock-dominated), through Ganymede and Califsto (water 1ce and rock) to Titan and Triton (uncertain composition but possibly like Ganymede with an addition of at least some more volatile ices, especially $\left(\mathrm{CH}_{4}\right)$. In the more ice-rich satellites, the structure is usually believed to be a rock-rich core surrounded by an ice layer that is partially or entirely solid [5]. The temperature ( $\$ 300 \mathrm{~K}$ ) and pressure (sfew tens of kilobars) encountered in this ice require detailed consideration of the water ice, $\mathrm{NH}_{3}-\mathrm{H}_{2} \mathrm{O}$ and $\mathrm{CH}_{4}-\mathrm{H}_{2} \mathrm{O}$ phase diagrams, some aspects of which are discussed further below. Titan is believed to have a hydrocarbon ocean [6] and Triton may have liquid $\mathrm{N}_{2}$ on its surface [7].

\section{IMPORTANT CONDENSED MATTER PROBLEMS}

We turn from these generalities about planets to a consideration of the material properties required for an improved understanding of planetary interiors. The following Table identifies the important pure constituents and mixtures; the subsequent discussion summarizes the current state of knowledge and identifies the work needed for further progress. 
Table II

Planetary Materials and Problems

\begin{tabular}{|c|c|c|}
\hline Material & Thermodynamic Conditions & Problem \\
\hline $\mathrm{H}_{2}$ & $P \leq 1$ Mbar, $T \leq 10^{4} \mathrm{~K}$ & dissociation, electrical \\
\hline $\begin{array}{l}\mathrm{H}-\mathrm{He} \\
\mathrm{H}_{2} \mathrm{O}\end{array}$ & $\begin{array}{l}P \sim \text { few Mbar, } T \sim 10^{4} \mathrm{~K} \\
100 \text { kbar } \& P \leqslant 5 \text { Mbar, } T \leq 5000 \mathrm{~K}\end{array}$ & $\begin{array}{l}\text { solubility, volume of mixing } \\
\text { nature of bonding (lonic?), } \\
\text { decomposition?, electrical } \\
\text { conductivity }\end{array}$ \\
\hline $\mathrm{H}_{2}-\mathrm{H}_{2} \mathrm{O}$ & same conditions & $\begin{array}{l}\text { solubility, fonization, } \\
\text { dissociation, conductivity }\end{array}$ \\
\hline $\begin{array}{l}\mathrm{H}_{2}-\mathrm{CH}_{4} \\
\mathrm{H}_{2}-\mathrm{CH}_{4}-\mathrm{CO}\end{array}$ & $\begin{array}{l}\text { same conditions } \\
\text { same conditions }\end{array}$ & $\begin{array}{l}\text { solubility, dissociation } \\
\text { dissociation, solubility, } \\
\text { clathrates, chemistry? }\end{array}$ \\
\hline $\begin{array}{l}\mathrm{NH}_{3}\langle-\rangle \mathrm{N}_{2}, \mathrm{H}_{2} \\
\text { Noble gases }\end{array}$ & 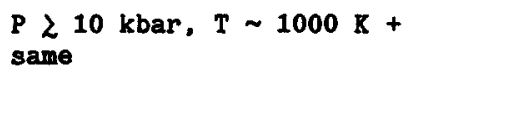 & $\begin{array}{l}\text { dissociation } \\
\text { solubility in metals, } \\
\text { partitioning between phases, } \\
\text { xenology }\end{array}$ \\
\hline
\end{tabular}

This table is not exhaustive and tends to emphasize binary endmembers of the real multicomponent system. The emphasis is on the major constituents, except for the entry on noble gases which has been included because these minor constituents may be important tracers of internal processes and (1n the particular case of xenon) may even have chemistry.

Molecular Bydrogen. The behavior of $\mathrm{H}_{2}$, especially at $\mathrm{P} 20.5 \mathrm{Mbar}$, is far from well understood. Simple models for dissociation and electronic excitation suggest a substantial change in the specific heat and Gruneisen $\gamma$, which may have a dramatic effect on the dynamics and evolution of Jupiter and Saturn. Perhaps the most interesting relevant experiment or theory for $\mathrm{B}_{2}$ concerns the value of the band gap energy between valence and conduction levels as a function of pressure, since this affects estimates of both thermodynamic and transport properties, especialiy electrical conductivity. The latter is important, since recent magnetohydrodynamic calculations [8] suggest that the observed wind profiles in the atmospheres of Jupiter and Saturn may be partly detêrmined by the electrical conductivity of $\mathrm{H}_{2}$ in the region where $\mathrm{p} \sim 0.2 \mathrm{~g} . \mathrm{cm}^{-3}$ and $\mathrm{T} \sim 5000 \mathrm{~K}$. Existing band structure calculations [9] indicate that the band gap in $\mathrm{H}_{2}$ decreases to zero at $P$ $\sim 1$ Mbar (presumably a precursor to the $\mathrm{H}_{2} \rightarrow$ monatomic metallic hydrogen transition). Direct confirmation of this behavior (e.g. optical reflectivity studies) or indirect evidence of this behavior (e.g., electrical conductivity at high $T$ ) would be highly desirable.

Hydrogen-Helium Mixtures. In the molecular regime, an accurate equation of state for $\mathrm{H}_{2}$-He would provide a determination of the $\mathrm{H}_{2}$-He interaction potential, essential for constructing equations of state for planetary interiors. Currently, this potential is estimated by theoretical arguments alone. However, the most important problem concerns the solubility of hellum in metallic hydrogen. Although this system is well understood in the infinite pressure limit [10] and moderately well understood in the low electron density limit [11], the phase diagram is least well understood in precisely the pressure range of most interest ( 1 to 10 Mbars). An appropriate theoretical calculation would involve evaluation of the "immersion energy": the energy cost of inserting a helium atom in the electron gas 
appropriate to metallic hydrogen. A Wigner-Seltz or density-functional approach would be appropriate.

Water. At high pressure $(P \sim 0.2-0.6 \mathrm{Mbar}), \mathrm{H}_{2} \mathrm{O}$ appears to either ionize as $\mathrm{H}_{3} \mathrm{O}^{+} \mathrm{OH}^{-}$if the temperature is high $(\mathrm{T} 21500 \mathrm{~K})[12,13]$ or "polymerize" (i.e. hydrogen bond is no longer distinguishable from a covalent bond) if the temperature is low $(T \sim 300 \mathrm{~K})$ [14]. It is clear that the range of possible behavior is large: further understanding of this is essential. Water should eventually metalize and might even decompose or disproportionate at sufficiently high pressure. None of these possibilities has been analyzed yet. One possible measurement of interest would be the optical band gap. This would provide information on electrical conductivity (even though the smallest band gap may be indirect) and may aid determination of the metallization pressure; both very important for improved of Uranus and Neptune and their probable magnetic fields.

Water-Hydrogen. Recent low pressure ( $P \leq 3 \mathrm{kbar}$ ) measurements [15] indicate that water and hydrogen mix in all proportions for $T 2650 \mathrm{~K}$. It is not known whether this behavior extends to very high pressures. If it does, then models for the deep atmosphere of Uranus [16] suggest a water cloud base at $P \sim 1$ to 2 kbar, below which a uniform $\mathrm{H}_{2}-\mathrm{H}_{2} \mathrm{O}$ mixture $\left(50-75 \% \mathrm{H}_{2} \mathrm{O}\right.$ by mass) extends to indefinite depths. An interesting consequence of this water-rich, hydrogen-poor environment is the finite equilibrium abundance of $\mathrm{N}_{2}$ (at the expense of $\mathrm{NH}_{3}$ ) and $\mathrm{CO}$ (at the expense of $\mathrm{CH}_{4}$ and $\mathrm{H}_{2} \mathrm{O}$ ), unlike the deep atmospheres of Jupiter and Saturn where $\mathrm{CH}_{4}$ and $\mathrm{NH}_{3}$ are the overwhelmingly preferred forms of carbon and nitrogen, respectively. In any event, it is very important to understand the $\mathrm{H}_{2} \mathrm{O}-\mathrm{H}_{2}$ phase diagram to extreme pressures. It is possible, for example, that this mixture is analogous to metal-ammonia (or alkali metal-alkali halide) mixtures at extreme pressure? A metallic state such as this may exist at a pressure substantially less than that needed to metallize pure water. As a side issue, it would also be of interest to know the solubility of He in $\mathrm{H}_{2} \mathrm{O}$ since the system of interest is, in reality, approximated as the ternary mixture $\mathrm{H}_{2}-\mathrm{He}^{-}-\mathrm{H}_{2} \mathrm{O}$.

Methane-Hydrogen. There is clear evidence that many hydrocarbons decompose (or collapse) upon shock compression, probably into graphite and hydrogen $[17,18]$. It is very important to establish the range of temperature and C:H ratios for which this decomposition can occur. It is equaliy important to establish whether an actual phase separation occurs (Implying possible formation of a diamond or liquid metallic (?) carbon layer in Uranus and Neptune) or whether a collapsed but intimately mixed $\mathrm{C}-\mathrm{H}$ structure results. Hugoniot data alone are insufficient to answer this question. Diamond cell work, perhaps at elevated temperatures, would seem most appropriate, Initially on pure methane or some other (saturated) hydrocarbon. There is already evidence that unsaturated bonds do not persist at high pressure [19].

Methane-Water. Pure methane ice does not condense under solar nebula conditions until $\mathrm{T} \leq 20 \mathrm{~K}$ and it is questionable whether these very cold conditions were ever achieved. However, the nearly stoichlometric clathrate hydrate $\left(\mathrm{CH}_{4} \cdot 5.75 \mathrm{H}_{2} \mathrm{O}\right.$, if ail cage sites are filled) forms more readily (at $\mathrm{T} \sim 40 \mathrm{~K}$ in the solar nebula, perhaps at $T \sim 80-100 \mathrm{~K}$ in the nebulae surrounding proto-giant planets). Remarkably little is known about clathrate compounds at high pressure, except for the tetrahydrofuran clathrate [20], probably a poor analog for the $\mathrm{CH}_{4}$ clathrate because of its different structure. A recent, detailed statistical mechanical treatment of clathrates [21] predicts that the methane clathrate decomposes at $\mathrm{P} \sim 12$ to $14 \mathrm{kbar}$ at all $\mathrm{T} \leqslant 300 \mathrm{~K}$ into ice VI and solid $\mathrm{CH}_{4}$. (At higher $T$, it decomposes at lower P.) This may have profound implications for the thermal histary of Titan, perhaps offering an explanation for the origin of $\mathrm{CH}_{4}$ from which the present ethane-rich ocean is derived [5,6]. A high priority experiment, probably accessible even with piston apparatus, is the stability of methane clathrate under pressure. 
At higher $T$ and $P$, it is of interest to know the mixing properties and chemical equilibria of the $\mathrm{CH}_{4}-\mathrm{H}_{2} \mathrm{O}$ system. What are the equilibrium abundances of $\mathrm{Co}, \mathrm{H}_{2}, \ldots$ ? Is phase separation possible in the deep interior, where water ionizes but $\mathrm{CH}_{4}$ remains neutral?

Nitrogen. At high pressure and temperature, it is known that $\mathrm{NH}_{3}$ is a good ionic conductor [13], presumably because of the formation of $\mathrm{NH}_{4}^{+} \mathrm{NH}_{2}^{-}$pairs. However, It may also be possible to form a fintte amount of $\mathrm{N}_{2}$; a process which is known to occur at low pressures in shock tubes. This may be of importance for the origin of molecular nitrogen in Titan and possibly Triton. The important point to stress is that despite the very high abundance of hydrogen in the outer system, it is possible to encounter conditions in which small but significant amounts of $\mathrm{N}_{2}$ are produced and preserved. High pressure and temperature processes are a likely example. The recently detected anomalous behavior of $\mathrm{N}_{2}$ under shock compression [22], suggesting dissociation or even metallization, raises interesting questions about the behavior of $\mathrm{N}-\mathrm{H}$ mixtures at megabar pressures.

Noble Gases. These are included here because noble gases are often used as tracers of processes in planetary interiors. For example, the outgassing of ${ }^{3} \mathrm{He}$ from Earth is sometimes used as an indicator of a "primordial reservoir" of material. An interesting question for the noble gases concerns the behavior of xenon. This element is more chemically reactive than the other noble gases and is believed to undergo an insulator-metal transition at $P \sim 1.3 \mathrm{Mbar}$ [23]. It is also "depleted" in the Earth's atmosphere, in the sense that $\mathrm{Xe} / \mathrm{Kr}$ (for example) is less on Earth than in meteorites. The cause of this is unknown but may be related to the high pressure properties of Xe. It is interesting to speculate whether analogous anomalies will arise when the Galileo probe (containing a mass spectrometer) enters Jupiter's atmosphere in the late 1980 's and measures the noble gas abundances.

\section{CONCLUDING COMMENTS}

The emerging perspective of planetary interiors suggests complexity and chemistry of a variety seldom suspected a decade ago. The 11ght molecular materials which predominate in the outer system exhibit a startling richness of behaviors which are becoming increasingly evident from shock compression and dlamond anvil cell experiments. In some respects, extreme pressure creates simplicity (e.g. everything eventually metalilizes) but planetary conditions include a far more complicated intermediate regime where dissociation, phase separation, and semiconduction are possible, probably likely. The exciting prospect of relating external observations to conditions deep within the planet (the outer planet analog of petrology) leads one to hope that the high pressure physics community continue to be aware of the Important applications of their work to planets.

\section{REFERENCES}

1. STEVENSON, D.J., Mat, Res, Soc, Symp, Proc,, 22, (1984), 357.

2. ZHARKOV, V.N. and TRUBITSYN, V.P.. Physics of Planetary Interiors (Publ. Pachart, Tucson, Arizona, USA), $1978,388 \mathrm{pp}$.

3. HUBBARD, W.B., Planetary Interiors (Publ. Van Nostrand Reinhold, New York), $1984,334 \mathrm{pp}$.

4. HUBBARD, W.B. and STEVENSON, D.J., In Saturn, ed. T. Gehrels (publ. Un. Arlzona Press, Tucson, Arizona). 1984, p. 47.

5. HUNTEN, D.M., TOMASKO, M.G., FLASAR, F.M., SAMUELSON, R.E., STROBEL, D.F. and STEVENSON, D.J., In Saturn, ed. T. Gehrels (pub1. Un. Arizona Press, Tucson, Arizona), 1984, p. 671 .

6. LUNINE, J.I., STEVENSON, D.J. and YUNG. Y.L., Science, 222, (1983), 1229.

7. CRUIKSHANK, D.P., BROWN, R.H, and CLARK, R.N.. Icarus, 58, (1984), 293.

8. KIRK, R.L. and STEVENSON, D.J., Bull, Am, Astron, Soc., 15, (1983), 825. 
9. FRIEDLI, C. and ASHCROFT, N.W., PhYs, Rev,, 16B, (1977), 662 .

10. STEVENSON, D.J., Phys, Rev., $128(1975), 3999$.

11. STBVENSON, D.J., J, Phys, F, Metal Physics, (1979), 791.

12. HAMANN, S.D. and LINTON, M., Trans, Faraday Soc., (GB) 62 (1966), 2234.

13. MITCHELL, A.K. and NELLIS, W.J., J Chem, Phys, 76, (1982), 6273.

14. POLIAN, A. and GRIMSDITCH, M., PhYs, ReV, Lett., 52 (1984), 1312.

15. SEWARD, T.M. and FRANCR, E.U., Ber, Bunsenges Phys, Chem, 85 (1981), 2.

16. STEVENSON, D.J., Bull, Am, Astron, Soc, 15 (1984), in press.

17. REE, F.H., J. Chem, Phys,, 70 (1979), 974.

18. ROSS, M., Nature, 292 (1981), 435.

19. NICOL, M., this conference.

20. ROSS, R.G. and ANDERSSON, P., Can, J, Chem,, 60 (1982), 881.

21. LUNINE, J.I. and STEVENSON, D.J.. Astrophys, J, Suppl, (1985) in press.

22. NELLIS, W.J., HOLMES, N.C., MITCHELL, A.C. and VAN THIEL, M., submitted to Phys, Rev, Lett. (1984).

23. ROSS, M. and McMAHAN, A.K., Phys, Rev,, 218 (1980), 1658. 